\title{
EULAR register of patients on immunosuppressive drugs
}

\author{
A $\mathrm{KAY}^{*}$ \\ From the ARC Epidemiology Research Unit (Clinical Section), Department of Rheumatology, Guy's \\ Hospital, London SE1
}

A collaborative United Kingdom/Australasian study of cancer in 5172 patients treated with immunosuppressive drugs ${ }^{115}$ showed an excess of non-Hodgkin's lymphoma, squamous cell skin cancer, and possibly mesenchymal tumours, in a series of patients with and without transplants. The 1349 non-transplant patients in the series, which included 492 with rheumatoid arthritis or ankylosing spondylitis, showed less marked excesses of the same tumours. In other studies, leukaemias have been reported in non-transplant patients exposed to cyclophosphamide. ${ }^{107}$

The purpose of the European League Against Rheumatism (EULAR) Study is to register and follow-up a substantial number of patients with connective tissue diseases who are, or have been, treated with immunosuppressive drugs, in order to determine the subsequent malignancy rate.

Seventy-seven rheumatologists $\dagger$ in 13 member countries registered 716 patients treated with immunosuppressive drugs between January 1979 and December 1980: registration of patients continues. The 478 patients who started immunosuppressive treatment on or after 1 January 1979 form a complete prospective sample. The 238 patients already taking immunosuppressives on 1 January 1979 are an incomplete sample but include patients with a longer drug exposure who may provide useful 'early-warning' information.

Of the 716 patients, 220 are male and 494 are female (two sex not stated). The mean age of the patients at the time of registration was 55 years (range six to 85). The conditions for which immunosuppressive drugs were given are shown in table 1. Azathioprine was the most commonly used drug (table 2) except in the treatment of polyarteritis nodosa, for which cyclophosphamide was more frequently used (table 3 ).

Seven malignancies have been reported so far (table 4) but it is not possible yet to estimate the malignancy rate. Follow-up of the patients continues

*On behalf of EULAR Standing Committee on International Clinical Studies and Therapeutic Trials. and the first full analysis of the findings will be undertaken in 1983.

TABLE 1 Conditions for which immunosuppressive drugs were given

\begin{tabular}{llr}
\hline & $\begin{array}{l}\text { No of patients } \\
\text { on register }\end{array}$ & $\%$ \\
\hline Rheumatoid arthritis (RA) & 581 & 81 \\
Systemic lupus erythematosus (SLE) & 35 & 5 \\
Psoriatic arthropathy (PsA) & 20 & 3 \\
Polyarthritis undefined & 11 & 2 \\
Polyarteritis nodosa (PAN) & 7 & 1 \\
Polymyositis (Pm) & 7 & 1 \\
Polymyalgia & 5 & 1 \\
Other & 40 & 6 \\
\hline
\end{tabular}

TABLE 2 Immunosuppressive drugs and frequency of usage: number of patients and (percentage)

\begin{tabular}{lccc}
\hline Drugs & $\begin{array}{l}\text { Prospective } \\
\text { sample }\end{array}$ & $\begin{array}{l}\text { Retrospective } \\
\text { sample }\end{array}$ & All patients \\
\hline Azathioprine & $288(60 \%) \ddagger$ & $159(67 \%)$ & $447(62 \%)$ \\
Cyclophosphamide & $105(22 \%)$ & $50(21 \%)$ & $155(22 \%)$ \\
Chlorambucil & $39(8 \%)$ & $27(11 \%)$ & $66(9 \%)$ \\
Proresid & $36(8 \%)$ & 0 & $36(5 \%)$ \\
Methotrexate & $9(2 \%)$ & $2(1 \%)$ & $11(2 \%)$ \\
Unknown & 1 & & 1 \\
\hline
\end{tabular}

$\ddagger$ Three patients were treated with azathioprine combined with another immunosuppressive drug ( 1 cyclophosphamide, 1 chlorambucil, 1 proresid).

TABLE 3 Immunosuppressive drugs used in relation to main conditions treated (see table 1 for abbreviations)

\begin{tabular}{lrrrll}
\hline & $R A$ & $S L E$ & $P S A$ & $P A N$ & $P m$ \\
\hline Azathioprine & 368 & 28 & 12 & 1 & 3 \\
Cyclophosphamide & 132 & 5 & 1 & 6 & 2 \\
Chlorambucil & 61 & 0 & 0 & 0 & 0 \\
Proresid & 33 & 0 & 1 & 0 & 0 \\
Methotrexate & 0 & 1 & 5 & 0 & 1 \\
\hline
\end{tabular}

TABLE 4 Type of malignancy reported in relation to diagnosis, sex, and immunosuppressive drugs

\begin{tabular}{llll}
\hline Diagnosis & Sex & Drug & Tumour \\
\hline RA & F & Cyclophosphamide & Acute myeloid leukaemia \\
RA & M & Azathioprine & Reticulum cell sarcoma \\
RA & M & Azathioprine & Ca bronchus (oat cell) \\
RA & M & Azathioprine & Ca bronchus \\
RA & F & Azathioprine & Ca cervix \\
RA & F & Azathioprine & Ca head of pancreas \\
\hline
\end{tabular}


$\doteqdot$ CONTRIBUTORS (1979 AND 1980)

Dr Bastin (Belgium)

Dr Brath (Norway)

Dr Bruckner (UK)

Dr Capell (UK)

Dr Cochrane (UK)

Dr Crouzet (France)

Dr Essigman (UK)

Professor Franchimont (Belgium)

Dr Gibson (UK)

Dr Golding (UK)

Professor Gullberg (Sweden)

Dr Haslock (UK)

Dr Hilton (UK)

Dr Huber-Bruning (Holland)

Professor Jayson (UK)

Professor Keitel (DDR)

Dr Mattingley (UK)

Dr Myles (UK)

Dr Nilson (UK)

Professor Panayi (UK)

Dr Rosenthal (Switzerland)

Dr E E Smith (UK)

Dr Stojanovic (Yugoslavia)

Dr Thompson (UK)

Dr Wiik (Denmark)

Dr Wysocka (Poland)
Dr Beardwell (UK)

Dr Brewis (UK)

Dr Budimir (Yugoslavia)

Dr Cardoe (UK)

Dr Colston (UK)

Dr Darlington (UK)

Professor Fehr (Switzerland)

Professor Frizziero (Italy)

Dr Glick (UK)

Dr Grahame (UK)

Dr Haavik (Norway)

Dr Helin (Denmark)

Dr Holden (UK)

Dr Huge (DDR)

Dr Jenni (Switzerland)

Dr Klafkl (DDR)

Dr Mennet (Switzerland)

Dr Newton (UK)

Dr Nissila (Finland)

Dr Popert (UK)

Dr Schwarz (Switzerland)

Dr P J Smith (UK)

Dr Sydnes (Norway)

Dr Webb (UK)

Dr Wojtulewski (UK)

Dr D Yates (UK)
Dr Boyle (UK)

Dr M Brown (UK)

Dr Camp (UK)

Dr Carty (UK)

Dr Corbett (UK)

Professor Deshayes (France)

Dr Feldman (UK)

Professor Gerber (Switzerland)

Dr Glyn (UK)

Dr Griffiths (UK)

Dr Hansen (Denmark)

Dr Hill (UK)

Dr Holt (UK)

Dr Jacoby (UK)

Professor Kahn (France)

Dr Kojander (Finland)

Dr Munthe (Norway)

Dr Nicholls (UK)

Professor Orloff (Belgium)

Dr Remans (Belgium)

Dr Sharp (UK)

Dr Steinberg (UK)

Dr Symons (UK)

Dr Webley (UK)

Dr Woodland (UK) 